\title{
Influence of fluorination time on surface flashover of epoxy in insulating gas
}

\author{
A. Mohamad* and G. Chen \\ Tony Davies High Voltage Lab \\ University of Southampton \\ United Kingdom \\ *a.mohamad@soton.ac.uk
}

\author{
B. X. Du \\ School of Electrical Engineering and Automation \\ Tianjin University \\ Tianjin, China
}

\begin{abstract}
The accumulation of charges on polymeric insulation under high voltage DC stress is a major concern in the transmission system as its formation distorts the local electric field and has an adverse impact on the electrical performance. By undergoing fluorination treatment on the insulation, improved surface properties similar to fluoropolymers can be attained without changing the bulk properties of the original material. The change in chemical components at the surface of polymeric insulation should lead to a corresponding change in electrical properties at the surface and thus can suppress charge build up. Previous research on surface flashover on fluorinated epoxy resin at atmospheric condition shows a significant improvement in flashover strength. In this paper, the authors investigated the effect of different degree of fluorination treatment on cast epoxy resin on suppressing surface charge accumulation and its contribution towards the improvement in surface breakdown strength. In comparison to previous research, it has been found that there is an optimum time duration of fluorination treatment which improves the surface breakdown strength of epoxy spacer. The mechanisms that govern the effect of fluorination treatment on surface flashover were discussed.
\end{abstract}

Keywords-fluorination; epoxy resins; flashover; EDX; nitrogen

\section{INTRODUCTION}

The triple junction interface of conductor-spacer-gas in high-voltage DC gas insulated switchgear (GIS) is known to be the most susceptible point for flashover to occur because it is the focal spot for charge injection [1]. Its importance has been highlighted in controlling and limiting charge accumulation on the surface of cast epoxy spacers under prolonged high-voltage DC stress. An essential aspect of surface charge accumulation is thought to concern the interface of the conductor, spacer surface and insulating gas. For this reason, a suitable modification in this particular area is believed to have a significant effect on suppressing charge accumulation on the spacer surface and so limiting the flashover occurrences inside GIS.

Recent studies tend to focus on modifications of bulk polymeric insulation, which lead to improvements in the properties of the bulk, as well as the surface layer. However, too little attention has been given towards modifications limited to surface properties alone by directly treating the surface layer of polymeric insulation. By applying chemical treatment on the surface of polymeric insulation, the surface charge transport characteristics of the polymeric material can be modified. The chemical treatment that is being used throughout this research is fluorination process, i.e. treatment of polymeric materials with fluorine or fluorine-inert gas (nitrogen, helium etc.) mixtures. This treatment has been well established from basic concepts to industrial applications. It is one the effective approaches in enhancing materials in term of barrier properties, separation properties, thermal and chemical stability, as well as biocompatibility [2-4].

It is interesting to note that, through fluorination treatment, excellent surface properties similar to those of fluoropolymers can be attained without affecting the bulk properties of the original insulating material. The researches and applications of fluorination process so far are mostly focused on improving properties with regards to wettability, adhesion, chemical stability, barrier properties, biocompatibility, and grafting [5]. There has been little attention given to modification in dielectric properties. In fact, the modifications in the chemical components of polymeric insulation should also lead to corresponding modifications in dielectric properties of the insulating material [6].

In this paper, epoxy resin samples were prepared and treated with various degree of surface fluorinating conditions. The samples then were characterised by Energy-dispersive Xray (EDX) analysis to confirm the formation of fluorination layer on the top surface of the cast epoxy resin. DC surface flashover test was carried out inside a glass chamber filled with nitrogen gas at atmospheric condition in order to investigate the performance of the treated samples inside encapsulated system.

\section{EXPERIMENTAL DETAILS}

\section{A. Sample preparation and fluorination}

A mixture of Bisphenol-A type epoxy resin with anhydride hardener and imidazole accelerator (weight ratio $100: 90: 2$ ) were used throughout this study. These components were degassed inside vacuum oven $\left(10^{3} \mathrm{~Pa}\right.$ at $\left.40^{\circ} \mathrm{C}\right)$ for 30 minutes and another 10 minutes after mixing. Afterwards, this mixture was filled into a stainless steel mould and was then placed in the oven at $80^{\circ} \mathrm{C}$ for 4 hours for gelation process. The mould was again heated at $120^{\circ} \mathrm{C}$ for another 8 hours for post-cured 
process. This matrix system offers a pathway to a highmolecular-weight polymer that can combine thermoplastic processability with a robust mechanical behaviour, adhesion to a variety of active surfaces, attractive optical characteristics and an exceptional barrier to oxygen and other atmospheric gases.

To investigate the improved electrical performance of surface fluorinated epoxy resin, the cast samples were fluorinated with different time durations. Fluorination of the samples was done in a closed stainless vessel. After evacuation, the fluorine and nitrogen mixture of ratio 1:4 is pumped into the vessel. The mixture gas pressure in the vessel is maintained at 225 mbars and $55^{\circ} \mathrm{C}$. Five different fluorination times were done; 30 minutes, 60 minutes, 120 minutes, 180 minutes, and 240 minutes. After the reaction, the reactive gases were purged from the vessel with nitrogen.

\section{B. Energy Dispersive X-rays Analysis}

EDX utilizes the X-ray spectrum produced by a subject bombarded with a focused beam of electrons to achieve a localized chemical spectrum. Theoretically, all elements from atomic number 4 (Be) to $92(\mathrm{U})$ can be spotted [7]. Qualitative analysis, i.e. the determination of the peaks in the spectrum is relatively straightforward due to the minimalism of X-ray spectra. Quantitative analysis, i.e. the identification of the concentrations of the elements present involves assessing peak strengths for each element in the sample.
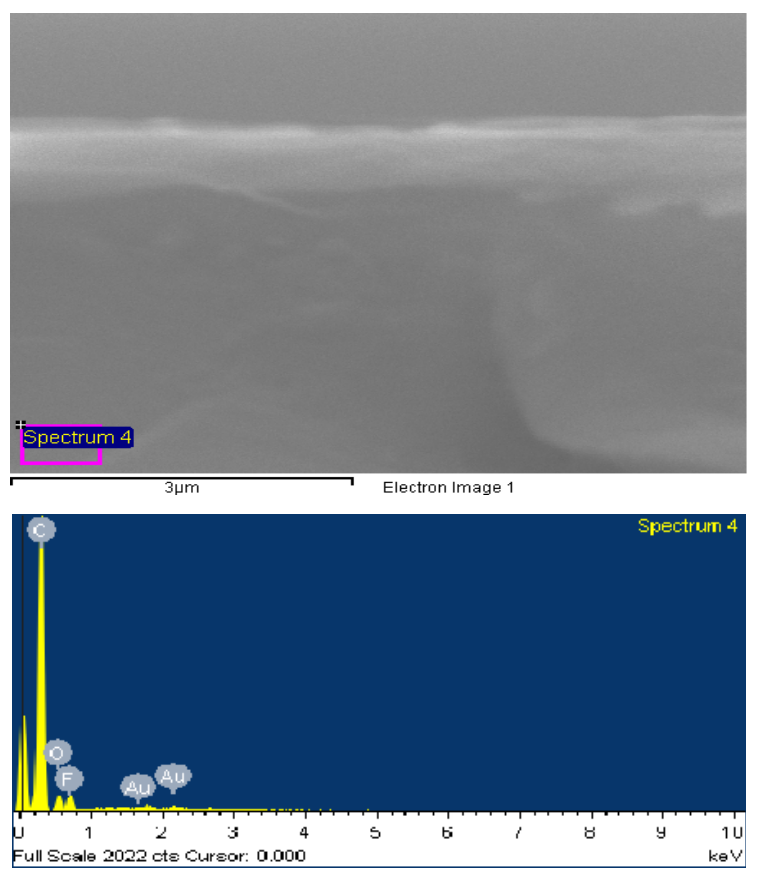

Fig. 1. The SEM image and EDX spectrum of the epoxy layer

The prepared samples were cut into small pieces of $10 \mathrm{~mm}$ $\mathrm{x} 10 \mathrm{~mm}$ and were gold coated before being put under the microscope. Fig. 1 and Fig. 2 show the scanning electron microscope (SEM) images and EDX spectrum of the crosssection of 60-minutes-fluorinated sample for epoxy layer and fluorinated layer respectively. Traces of gold (Au) can be seen from the spectrums due to the gold element from the gold coating of the samples. In Fig. 1, peaks of carbon (C) and oxygen $(\mathrm{O})$ can be seen from the EDX spectrum taken from the spot $3 \mu \mathrm{m}$ deep into the sample. These elements are the main elements for epoxy. However, another main element for epoxy, hydrogen, is not detectable due to the limitation of EDX in detecting elements of small atomic number. A tiny peak of fluorin (F) can be seen as well due to the existence of fluorinated layer near the vicinity of the EDX focus spot. The fluorin peak is greater in intensity in Fig. 2, indicating a higher concentration of fluorin element on the top layer as a product from the fluorination treatment. The level of fluorination depends upon the treatment parameters of the composition and pressure of the reactive mixture, fluorine partial pressure and treatment time, as well as temperature and nature of the polymer in use [8]. A Raman spectrum was conducted in authors' previous paper to prove that a chemical reaction did take place on the surface of epoxy [9].
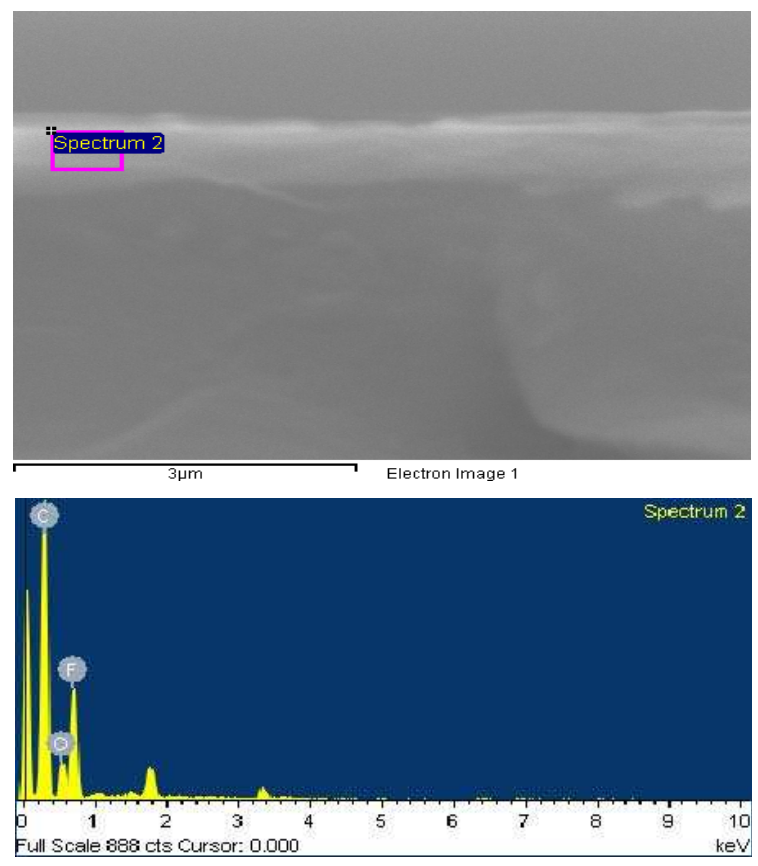

Fig. 2. The SEM image and EDX spectrum of the fluorinated layer

\section{Flashover in nitrogen gas test}

The electrode system is constructed in a typical surface breakdown arrangement as used by various authors [10-12] in which the breakdown is likely to occur along the surface between the triple junctions of electrode-sample-gas. This test apparatus is designed to study the surface flashover strength of fluorinated epoxy samples of different fluorination times inside a controlled environment. The schematic diagram of this test is shown in Fig. 3.

Both electrodes are made from tungsten. The edges of the electrodes were rounded to $4.5 \mathrm{~mm}$ radius while sockets for banana plug were bored at the rear of both electrodes. The base for the sample holder is made from Teflon. The gap between the electrodes is adjustable, and for this experiment, the gap was set at $8 \mathrm{~mm}$ in order to get field strength of $\sim 2 \mathrm{kV} / \mathrm{mm}$ during breakdown. $300 \mu \mathrm{m}$ thick epoxy resin samples were used, and they were placed in between the electrode pair and 
on the Teflon base. This electrode setup was put inside a glass chamber that was connected to a vacuum pump and nitrogen gas tank. The glass chamber was designed to provide maximum accessibility and visibility for imaging. A highspeed camera with the capability of capturing images at maximum 3000 fps was positioned in front of the viewing window of the glass chamber. The camera was focused on the $8 \mathrm{~mm}$ gap space between the tungsten electrodes.

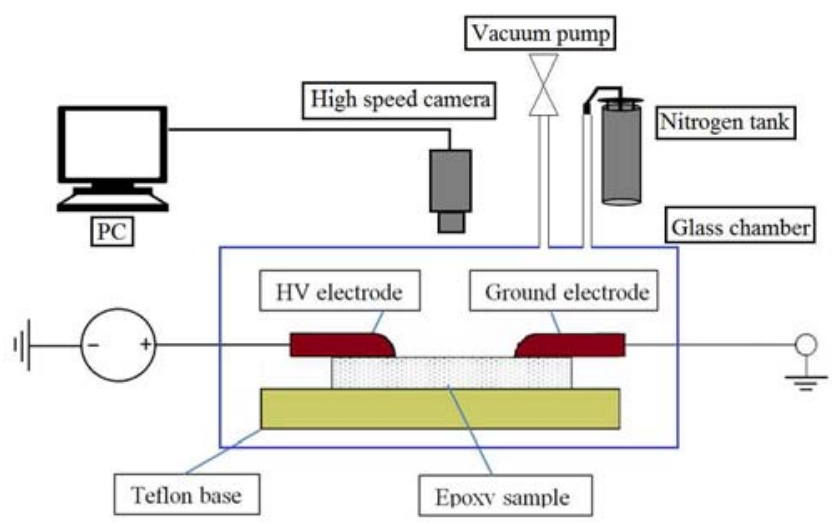

Fig. 3. Schematic diagram of surface DC flashover kit in nitrogen gas at atmospheric condition

The chamber was vacuumed to $10^{-1}$ mbar to remove the remaining moisture inside the chamber. Nitrogen gas was then pumped in through a desiccant filter in order to dry out the nitrogen and to minimise the humidity content inside the chamber. The samples were subjected to a linearly increased voltage of $100 \mathrm{~V} / \mathrm{s}$ until they underwent flashover. This flashover test was repeated 25 times for each degree of fluorination, and the results were plotted using Weibull distribution plot. It has been noted that, after a number of flashover occurrences, pitting signs could be seen on the edge of both tungsten electrodes. Because of this, the electrodes were re-polished after every five flashovers, in order to remove the pitting signs and thus more consistent results.

\section{RESULTS AND DISCUSSIONS}

The flashover performance of the epoxy resin samples can be described using two-parameter Weibull distribution analysis. Assuming that the random breakdown process follows the Weibull distribution, the graph of failure probability is plotted against the breakdown voltage where failure probability indicates the likelihood of the breakdown phenomenon in an applied field. The scale parameter, $\alpha$, represents the breakdown strength and the shape parameter, $\beta$, represents a measure of the spread of the breakdown data. The smaller value of $\beta$, the larger is the scatter of the breakdown data. The result in Fig. 4, in general, shows an increasing trend in DC surface breakdown strength with the introduction of fluorinated surface layer.

For non-fluorinated sample (F00), surface breakdown strength of a scale parameter $12.40 \mathrm{kV}$ is observed. The DC surface breakdown voltage increases as the fluorination time increases. The increase in breakdown voltage is marginal for the 30minute-fluorinated sample (F30). The highest DC breakdown scale parameter voltage is recorded on 180-minutes-fluorinted sample with surface breakdown voltage as high as $21.3 \mathrm{kV}$ and an improved shape parameter of 19.60. However, a drop in breakdown voltage for 240-minutes-fluorinated sample (F240) with a low shape parameter is observed.

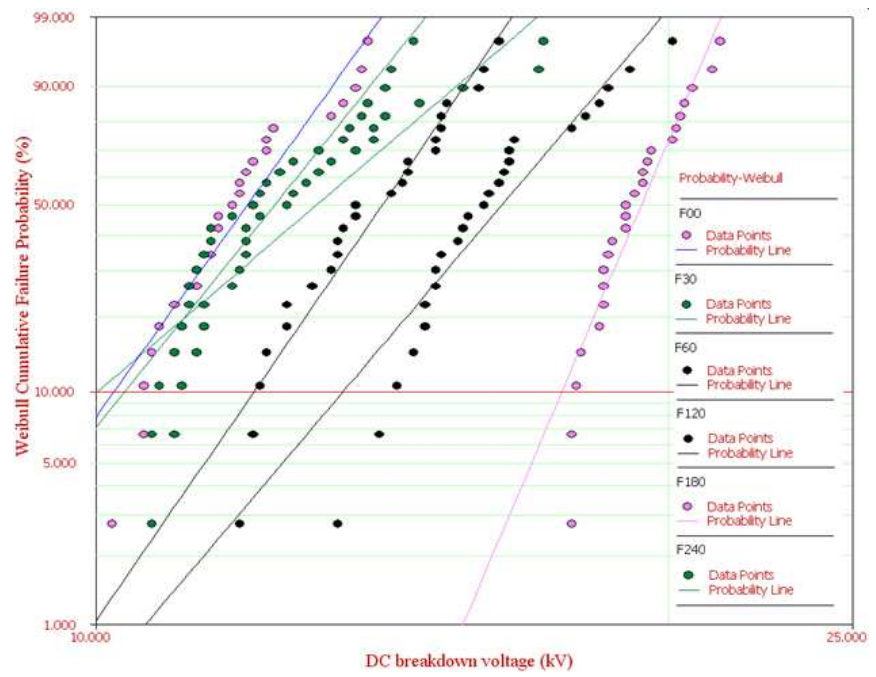

Fig. 4. Weibull distribution for $300 \mu \mathrm{m}$ epoxy resin samples of different fluorination time

TABLE I. WEIBULL PARAMETERS

\begin{tabular}{|c|c|c|}
\hline Sample & Scale parameter $(\boldsymbol{k}), \boldsymbol{\alpha}$ & Shape parameter, $\boldsymbol{\beta}$ \\
\hline F00 & 12.40 & 11.64 \\
\hline F30 & 12.86 & 10.37 \\
\hline F60 & 14.58 & 12.08 \\
\hline F120 & 16.97 & 9.80 \\
\hline F180 & 19.72 & 19.60 \\
\hline F240 & 13.76 & 7.09 \\
\hline
\end{tabular}

Surface charge accumulation, which leads to surface flashover, is believed to be triggered by a field emission that occurs at the sharp edges of the electrodes under high-voltage DC stress [13]. The emitted electrons from protrusions on the rough finish electrodes drift in the direction of the electric field to reach the epoxy resin surface. There are three possible mechanisms for surface flashover; (I) a micro-discharge at an imperfect contact at the triple junction interface, (II) a microdischarge at an imperfection in the epoxy resin sample surface, or (III) at the particle in proximity to the spacer surface [14]. These discharges act as high-field spots and cause rapid electron emission and ionisation. The generated charges are trapped on the insulator surface and further distort the local electric field and consequently initiated further ionisation processes. These micro-discharges could well develop into a path that leads to a flashover along the region on the surface where the distortion of electric field exceeds the threshold strength of the insulating surface.

With the incorporation of fluorinated substituent onto the surface of epoxy resin sample as confirmed by the EDX spectrum, the conductivity along the dielectric surface is 
increased [15]. The conductivity is higher with longer time duration of fluorination treatment. Interestingly, increase in surface conductivity enables trapped surface charges to leak away faster and, as a result, suppresses the surface charge accumulation from distorting the local electric field [16]. Hence, the increasing trend in DC surface flashover strength over fluorination time with the highest surface breakdown strength is observed in sample F180. At 180 minutes of fluorination time, the surface layer is at an optimum conductivity value which enables the generated surface charges to leak away from traps.

However, there is a limit to the increase in surface conductivity for which the surface breakdown strength can be enhanced. As evidenced in sample F240, in which at this point, the insulating surface has become too conductive and delivers an opposite effect towards the surface breakdown strength. Instead of dissipating the accumulation of generated surface charges from traps on top of the epoxy surface, the fluorination layer on sample F240 contributes towards the formation of breakdown path on the surface layer and effectively lowers the surface breakdown strength of the insulating material. At room temperature and insulating nitrogen gas at atmospheric pressure, the breakdown on the epoxy surface follows a Townsend mechanism of breakdown [17]. The increase in conductivity on the surface facilitates the ionisation process along the surface. The ensuing free surface charges are then accelerated by a high electric field and results in electrical conduction along the surface via avalanche multiplication from ionisation of molecules by ion impact. The author believe the same kind of phenomenon can be observed at gap other than $8 \mathrm{~mm}$ because the dissipation of surface charges work best at conductivity of 180-minutes-fluorinated sample at the same field strength.

\section{CONCLUSIONS}

This research was tailored to look at the result of the fluorination treatment on epoxy resin structure in enhancing the dielectric properties of the material for the use in highvoltage DC applications. The fluorinated epoxy resins had been characterised and electrically investigated to give a better understanding of its insulating performance.

The epoxy samples were characterised using SEM and EDX analysis in order to qualitatively and quantitatively determine the chemical composition of the treated samples. It has been proven that from the chemical treatment, the treated samples do form an additional layer on top of the cast epoxy resin that contains fluorin element that is visible from the EDX spectrum.

Finally, from the DC flashover test, there is an obvious trend of increasing surface breakdown strength with the increase in time duration of fluorination treatment. This is because the incorporation of fluorinated surface layer on epoxy resin slightly improves the conductivity along the surface. The improvement enables any trapped charges on the surface to leak away faster and, for this reason, suppresses the charges accumulation and minimizes distortion of the local electric field on epoxy resin surface. The highest value for surface breakdown strength is recorded from the 180-minutes- fluorinated sample that turns out to be the optimum duration for fluorination treatment. The results of this study show that the fluorination treatment on epoxy resin appears to play a significant role in improving the dielectric properties of epoxy resin.

\section{REFERENCES}

[1] T. Nitta and K. Nakanishi "Charge accumulation on insulating spacers for HVDC GIS" IEEE Transactions on Electrical Insulation Vol. 26 No. 3, pp 418-427, June 1991.

[2] A.P. Kharitonov "Direct fluorination of polymers - From fundamental research to industrial applications" Progress in Organic Coatings, vol. 61, issues 2-4, pp.192-204, February 2008

[3] A. Tressaud, E. Durand, C. Labrugere, A.P. Kharitonov, L.N. Kharitonova "Modification of surface properties of carbon-based and polymeric materials through fluorination routes: From fundamental research to industrial applications" Journal of Fluorine Chemistry, vol. 128, pp378-391, January 2007.

[4] Laurence W. McKeen "Fluorinated coatings and finishes handbook" William Andrew Publishing, Inc. pp. 1-10.

[5] Yue Jiang, Zhenlian An, Chenxia Liu, Feihu Zheng, Yewen Zhang "Influence of oxyfluorination time on space charge behavior in polyethylene" IEEE Transactions on Dielectrics and Electrical Insulation Vol. 17, No. 6, pp. 1814-1823, December 2010.

[6] Z. An, Q. Yang, C. Xie, Y. Jiang, F. Zheng, Y. Zhang "Suppression of charge injection to linear low density polyethylene by surface fluorination modification" International Symposium on Electrical Insulating Materials, pp.368-371, September 2008.

[7] University of California Riverside "Introduction to Energy Dispersive X-ray Spectrometry (EDS)" Central Facility for Advanced Microscopy and Microanalysis, pp. 1

[8] F.J. du Toit, R.D. Sanderson "Surface Fluorination of polypropylene:1. Characterisation of surface properties" Journal of Fluorine Chemistry, vol. 107, pp. 107-115, 1999

[9] A. Mohamad, G. Chen, Y. Zhang and Z. An "Surface Fluorinated Epoxy Resin for High Voltage DC Application" IEEE Transactions on Dielectrics and Electrical Insulation, Vol. 22, No.1, pp. 101-108, Feb 2015

[10] Cheng Rong Li, Li Jian Ding, Jin Zhuang Lv, You Ping Tu, Yang Chun Cheng "The relation of trap distribution of alumina with surface flashover performance in vacuum" IEEE Transactions on Dielectrics and Electrical Insulation, Vol. 13, No. 1, pp. 79-84, February 2006

[11] M. Serkan, H. Kirkici, K. Koppisetty "Surface flashover characteristics of nano particle cast epoxy resin" Pulsed Power Conference, pp. 950953, June 2005

[12] John T. Krile, Andreas A. Neuber, James C. Dickens, Hermann G. Krompholz "DC and pulsed dielectric surface flashover at atmospheric pressure" IEEE Transactions on Plasma Science, vol. 33, no. 4, pp. 1149-1154, August 2005

[13] A. Mohamad, G. Chen, Y. Zhang, Z. An "Influence of fluorination time on surface flashover of polymeric insulation" Conference on Electrical Insulation and Dielectric Phenomena (CEIDP), October 2013

[14] Alan H. Cookson "Electrical breakdown for uniform fields in compressed gases" Proceedings of the Institution of Electrical Engineers, vol. 117, issue 1, pp.269-280, January 1970

[15] A. Mohamad, G. Chen, Y. Zhang, Z. An "Mechanisms for surface potential decay on fluorinated epoxy in high voltage DC applications" Conference on Electrical Insulation and Dielectric Phenomena (CEIDP), October 2014

[16] A. Mohamad, G. Chen, Y. Zhang and Z. An "Surface potential decay measurements on fluorinated polymeric insulation for high-voltage DC applications" Conference on Electrical Insulation and Dielectric Phenomena (CEIDP), October 2013

P. Keith Watson and A. Harry Sharbaugh "The electric strength of nitrogen at elevated pressures and small gap spacings" Journal of Applied Physics, vol. 40, No. 1, pp. 328-336, January 1969 